\title{
Microfluidic Device for Controllable Chemical Release via Field-Actuated Membrane Incorporating Nanoparticles
}

\author{
Xiang Wang, ${ }^{1}$ Shunbo Li, ${ }^{2}$ Limu Wang, ${ }^{1}$ Xin Yi, ${ }^{1}$ Yu Sanna Hui, \\ Jianhua Qin, ${ }^{3}$ and Weijia Wen ${ }^{1,2}$ \\ ${ }^{1}$ Nanoscience and Nanotechnology Program, Hong Kong University of Science and Technology, Clear Water Bay, Kowloon, Hong Kong \\ ${ }^{2}$ Department of Physics and KAUST-HKUST Joint Micro/Nanofluidic Laboratory, Hong Kong University of Science and Technology, \\ Clear Water Bay, Kowloon, Hong Kong \\ ${ }^{3}$ Dalian Institute of Chemical Physics, Chinese Academy of Science, 457 Zhongshan Road, Dalian, China
}

Correspondence should be addressed to Weijia Wen; phwen@ust.hk

Received 2 July 2013; Accepted 25 August 2013

Academic Editor: Krasimir Vasilev

Copyright (C) 2013 Xiang Wang et al. This is an open access article distributed under the Creative Commons Attribution License, which permits unrestricted use, distribution, and reproduction in any medium, provided the original work is properly cited.

\begin{abstract}
We report a robust magnetic-membrane-based microfluidic platform for controllable chemical release. The magnetic membrane was prepared by mixing polydimethylsiloxane (PDMS) and carbonyl-iron nanoparticles together to obtain a flexible thin film. With combined, simultaneous regulation of magnetic stimulus and mechanical pumping, the desired chemical release rate can easily be realized. For example, the dose release experimental data was well fitted by a mathematical sigmoidal model, exhibiting a typical dose-response relationship, which shows promise in providing significant guidance for on-demand drug delivery. To test the platform's feasibility, our microfluidic device was employed in an experiment involving Escherichia coli culture under controlled antibiotic ciprofloxacin exposure, and the expected outcomes were successfully obtained. Our experimental results indicate that such a microfluidic device, with high accuracy and easy manipulation properties, can legitimately be characterized as active chemical release system.
\end{abstract}

\section{Introduction}

Controllable release describes materials or devices that can control the release time or the release rate of chemicals or both. This technique has provided broad usefulness in different fields such as foods, cosmetics, pesticides, and agricultural industries $[1,2]$, while the most important application is active drug release [3]. It is well known that how and when a drug is delivered can have a significant effect on its potency. Traditional drug-release systems are characterized by immediate and uncontrolled drug-delivery kinetics, such system is usually referred to as passive drug release. Under such circumstance, it is possible that a given drug concentration dangerously approaches its toxic threshold to subsequently fall below the level with effective potency. From the viewpoint of the pharmacotherapy optimization, drug release should be controlled in accordance with the potent purpose and the pharmacological properties of active substances. This purpose has given great impetus to the concept known as active drug release, which first arose in the 1960s. Typically, controlled or active release is used to achieve sustained or pulsatile drug release. The rationale is to maintain drug concentration in the target receptors at a desired value as long as necessary [4]. What is actively controlled, in other words, is the drug-release rate and duration [5]. Compared with conventional dosage protocols, controlled drug release systems offer numerous advantages such as enhanced efficacy and reduced toxicity [6].

In recent years, many of the thrusts into the field have spurred the rapid development of advanced drug-release systems and made numerous new discoveries. One popular approach involves incorporation of drug molecules into the matrix of microscopic polymer spheres or capsules [7, 8]. Most are fabricated with polymers having particular physical or chemical characteristics, such as good biodegradability, biocompatibility or sensitive responses to $\mathrm{PH}$ value 
[9-11], temperature [12], light intensity $[13,14]$, external electric [15] or magnetic field [16], glucose [17], and others. Additionally, recent achievements in microtechnologies have been applied in designing drug-release microdevices, for example, microneedles and implantable microchips [1820], which possess inherent advantages such as hand-held portability, sample saving, and implantable properties. The aforementioned controlled drug-release systems, moreover, can circumvent problems that are commonly encountered in traditional drug-release methods. However, some of these systems fail to achieve any desired complex release profile or involve complicated design and fabrication. To address these problems, we here introduce a novel prototypical microfluidic controlled-chemical-release platform based on a magnetic-field-actuated composite membrane which incorporates carbonyl-iron nanoparticles for actuation by magnetic field. Compared with existing release systems, our platform possesses such remarkable advantages as simple fabrication, easy manipulation, and high accuracy. And we demonstrate the microfluidic chip's feasibility for the realization of customizable release profiles as well as its general applicability in the drug release for biological system.

\section{Experiments, Results, and Analyses}

2.1. Materials. Polydimethylsiloxane (PDMS) was used in the fabrication of both the microfluidic chip and magnetic composite membrane due to its good flexibility, biocompatibility, and transparency, among other merits. The magnetic membrane used as the drug-delivery component, consists of carbonyl-iron (CI) nanoparticles mixed with PDMS, socalled CI-PDMS [21]. Due to its high magnetization and good mechanical elasticity, the CI-PDMS magnetic membrane shows good vibration characteristics under an external alternative magnetic field [21]. Ciprofloxacin and GFP recombinant $E$. coli bacteria in L-Broth standard growth medium, one liter of which contains $10 \mathrm{~g}$ tryptone, $5 \mathrm{~g}$ yeast extract, $10 \mathrm{~g}$ sodium chloride, and $2 \mathrm{~mL}$ ampicillin solution $(100 \mathrm{mg} / \mathrm{mL})$, were employed in the biological experimentation.

\subsection{Microfluidic Chip Fabrication. Figure 1(a) schematizes} the three-dimensional microfluidic chip fabrication procedure. It can be seen in the upper-left area of the figure, premixed PDMS gel (Sylgard 184 Silicone Elastomer, Dow Corning Corporation; weight ratio of prepolymer:crosslinker $=10: 1$ ) was mixed with CI nanoparticles and degassed, after which it was spun-coated on a Teflon-treated glass wafer so as to form thin layers of CI-PDMS. After solidification in an oven $\left(65^{\circ} \mathrm{C}, 2\right.$ hours), a CI-PDMS membrane of about $100 \mu \mathrm{m}$ in thickness was fabricated to the desired size using the laser-cutting technique. The CI-PDMS composite properties can be found in our previous report [21]. The chamber layers were patterned directly onto a cured PDMS sheet by laser cutting, as shown in the upper-right area of Figure 1(a). The aforementioned layers were bounded together by means of oxygen plasma bonding. The same steps were repeated for the fabrication of the diffusion PDMS membrane and the lower chamber layers. The microfluidic chip shown in the lower inset of the figure consists of two individual chambers separated by a diffusion membrane, while at the bottom of the chip, a CI-PDMS magnetic membrane is located. It should be pointed out that other hydrophilic polymer films (e.g., MEMBRA-CEL MC18 X 100 CLR, Viskase Companies, USA) also can be used as the diffusion membrane and that the bonding methodology is similar to that for PDMS thin film. Figures 1(b)-1(c) show the top and bottom views, respectively, of a well-made microfluidic chip including five reservoirs, each one with diameter of $1000 \mu \mathrm{m}$ and depth of $0.1 \mathrm{~cm}$.

2.3. Working Mechanism of Microfluidic Chip. The experimental setup shown in Figure 2 was used to test the release characteristics of the microfluidic chip. In the upper chamber, water was injected to the full; sodium chloride $(\mathrm{NaCl})$ solution of $0.5 \mathrm{~mol} / \mathrm{L}$ was pumped to the bottom chamber with flow rate $0.04 \mathrm{~mL} / \mathrm{h}$. Two platinum electrodes, mounted in the upper chamber and connected to a digital multimeter (Agilent 34411A), were used to determine the solution's conductance variation when the $\mathrm{NaCl}$ diffused from the lower chamber to the upper one through the diffusion membrane. An electromagnet positioned directly beneath the CI-PDMS membrane of the microchip served to actuate membrane vibration via push-pull movement when acted on by an alternative current within the $60-110 \mathrm{~Hz}$ frequency range, while two syringe pumps controlled the flow status of two fluids to the two chambers. LabVIEW software was utilized for device control and data collection.

\subsection{Determination of Chemical-Release Profiles. Figure 3(a)} presents the $\mathrm{NaCl}$ ion concentration's variation profiles resulting from the changes of electrical conductance in the upper chamber when the solution diffused from the lower chamber through the membrane. Based on the proportional relationship between conductance and ion concentration, the vertical axis is normalized to the original conductance and represents ion concentration. These profiles can be realized under different working conditions of the electromagnet and syringe pump 2 (in the current experiment, syringe pump 1 was used only for water filling and usually was kept in the OFF state). The ON/OFF signals for both the electromagnet and syringe pump 2 are illustrated in Figure 3(b). For example, when syringe pump 2 was $\mathrm{ON}$ and the electromagnet was OFF over the entire working duration, the solution diffused naturally through the membrane without any actuated function, the corresponding variation of the $\mathrm{NaCl}$ ion concentration delivery route in the upper chamber is drawn as curve 2. It can be seen that the magnetic field applied to the magnetic membrane obviously strengthens the diffusion effect caused by membrane vibration. $\mathrm{NaCl}$ diffused very rapidly initially and then slowly approached the saturation value. Other routes could also be obtained by varying the ON/OFF states of electromagnet and syringe pump 2. The demonstrated curves are plotted in Figure 3(a) and the corresponding actuated signals are shown in Figure 3(b). 


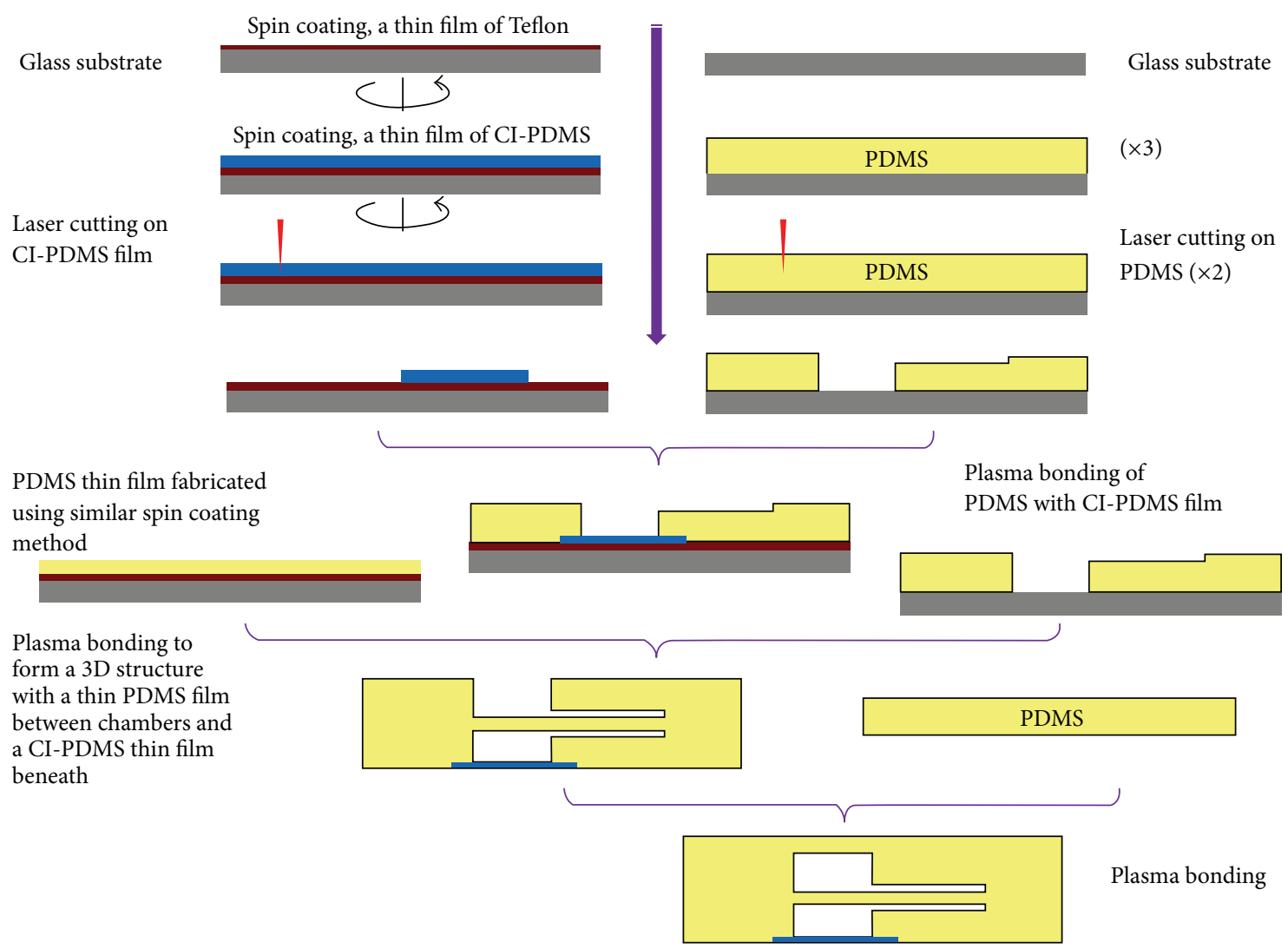

(a)

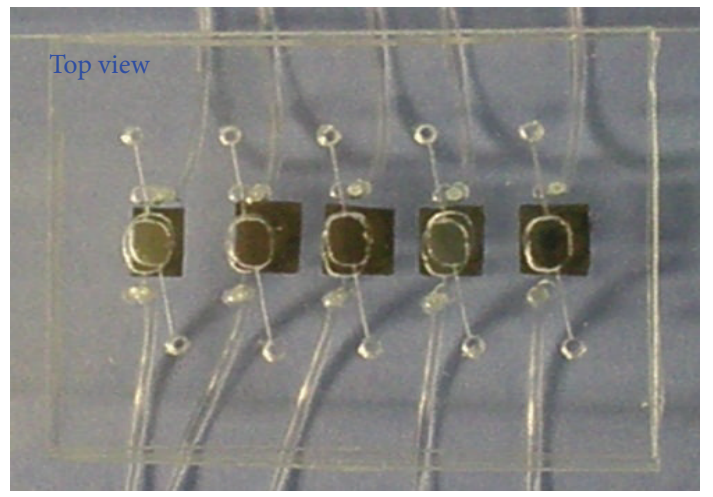

(b)

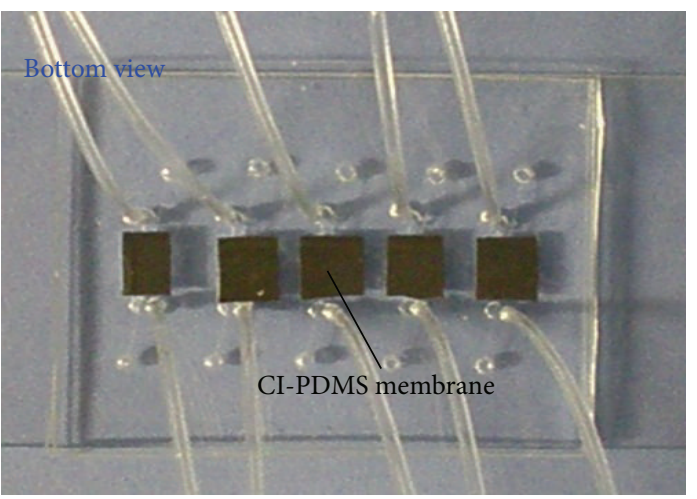

(c)

FIGURE 1: The preparation of drug delivery microfluidic chip and real pictures: (a) schematical fabrication process; (b) top view of this chip; (c) bottom view of this chip.

Sigmoidal fitting to the experimental data was carried out with the following Boltzmann equation of software OriginPro-8:

$$
y=\frac{A_{1}-A_{2}}{1+e^{\left(x-x_{0}\right) / d x}}+A_{2},
$$

where $y$ represents the ion concentration measured in the upper chamber, $A_{1}$ and $A_{2}$ are the initial and final values of the ion concentration, respectively, $x_{0}$ is the inflection point at which $y$ shows the most significant change, and $d x$ relates to the slope of the tangent line at $x_{0}$. These parameters are clearly illustrated in the upper-right inset of Figure 3(a).

Since the ion concentration was normalized to 1 as the maximum, $A_{1}$ and $A_{2}$ in (1) can be fixed to 0 and 1 , respectively. Thus, (1) can be rewritten as

$$
y=\frac{1}{1+e^{-\left(x-x_{0}\right) / d x}} .
$$

From (2), we can simply obtain $y^{\prime}\left(x_{0}\right)=1 / 4 d x$ and $y^{\prime \prime}\left(x_{0}\right)=0$. 


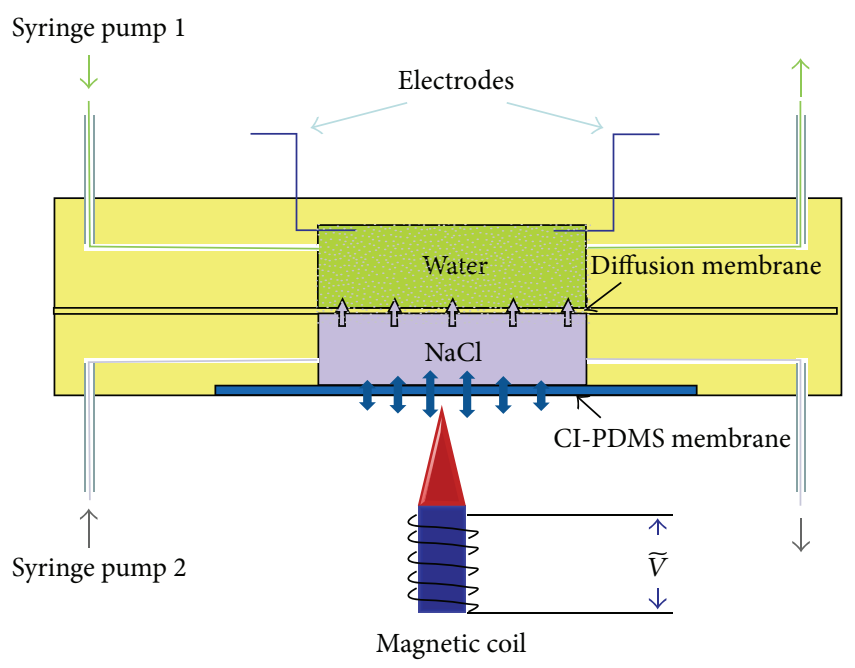

FIgURE 2: Cross-section view of the microfluidic device.

The first-order derivative of (2) at $x_{0}$ is inversely proportional to the parameter $d x$, the smaller value of which signifies the larger ion concentration changes per unit time. The inflection point $x_{0}$ marks the time at which the growth rate of the solution's diffusion effect of solution shifts from acceleration to deceleration. Therefore, the second-order derivative at $x_{0}$ equals zero.

Interpreting the parameters in their biological meanings is helpful in understanding the corresponding drugrelease characteristics. From a pharmacological standpoint, the $x_{0}$ value can be used to differentiate drug administration rates, acceleration or deceleration, the steepness factor $d x$ representing a most significant response per unit change in administered dose. Accordingly, these parameters can be utilized as indicators in evaluating the potency and efficacy of administrated drugs. If assigning any arbitrary values to $x_{0}$ and $d x$, we can, based on the solution diffusion results, purposely construct a new delivery route by proper, simultaneous manipulation of the respective magnetic field and pumping signals. In this way, active drug release can be achieved.

Figure 3(a) clearly shows good agreement between the fitting results from above mathematical model and the experimental data. Table 1 indicates that the values for $x_{0}$ and $d x$ differ from each other for different curves, which result from the combined regulation of the magnetic field and mechanical pumping. From the fitted value, we note that $d x$ and $x_{0}$ for curve 1 are much smaller than those for curve 2 .

Biological Experimentation for Dose-Response Assessment. The sigmoid-shaped curve in Figure 3(a) represents a typical dose-response relationship, which is the most fundamental and pervasive concept in toxicology [22]. Studying doseresponse model is crucial to determine the "safe" and "hazardous" levels and dosages of drugs to which organisms are exposed. It is known that dose- responses can be elucidated in cell culture preparations wherein there is a discrete and controlled pharmacologic environment. Additionally,
TABLE 1: Fitting parameters for curves 1-5 in Figure 3(a).

\begin{tabular}{lccccc}
\hline Parameter & \multicolumn{5}{c}{ Curve } \\
& 1 & 2 & 3 & 4 & 5 \\
\hline$x_{0}$ & 28.85 & 67.84 & 140.55 & 194.07 & 246.1 \\
$d x$ & 16.45 & 43.42 & 54.7 & 27.24 & 16.98 \\
\hline
\end{tabular}

a comparison of dose-response curves among drugs can identify both the therapeutic and the toxic effects of the potential drugs. Thus, given the above experimental results, we deduced that if we substituted, in a biological experiment, ciprofloxacin solution and growth medium (L-Broth) for sodium chloride and water, respectively, similar doseresponse consequences could be observed. Ciprofloxacin is a synthetic chemotherapeutic antibiotic of the fluoroquinolone drug class $[23,24]$, while $E$. coli is a gram-negative rodshaped bacterium commonly found in the lower intestine of warm-blooded organisms (endotherms). Pharmacologically, ciprofloxacin can inhibit the reproduction and genetic material repairment of GFP recombinant $E$. coli bacteria.

In order to verify our hypothesis and characterize the established drug-delivery platform for realistic application, ciprofloxacin was pumped into the lower chamber and GFP recombinant $E$. coli bacteria with L-Broth standard growth medium in the upper compartment. One liter of this medium contains $10 \mathrm{~g}$ tryptone, $5 \mathrm{~g}$ yeast extract, $10 \mathrm{~g}$ sodium chloride, and $2 \mathrm{~mL}$ ampicillin solution $(100 \mathrm{mg} / \mathrm{mL})$. Similar to the microfluidic chip shown in Figure 2, the chip used in the present experiment consisted of five pairs of reservoirs labeled C1, C2, C3, C4, and C5, respectively. The drugdelivery routes of ciprofloxacin for $\mathrm{Cl}-\mathrm{C} 5$ were consistent with curves 1-5 described in Figure 3(a), but the experiment was carried out for $120 \mathrm{~min}$. During the experiment, fluorescent images of $E$. coli in the chip reservoirs were taken by a Canon camera (EOS 5D, Mark II) equipped with a macro lens.

We extracted the average light intensity from each optical image using Adobe Photoshop software, and the results are illustrated in Figure 4, where it is evident that in chamber C5, the E. coli grew and multiplied very well. The intensity of fluorescence doubled every $20 \mathrm{~min}$ during the first one and a half hours, since initially less drug amount was diffused to the cell chamber. Thereafter, the growth rate decreased somewhat. The reason lies in the limitation of L-Broth growth medium; that is, it becomes deficient after one-hour cell culture; consequently, the drug effect increased more rapidly. With regard to chamber $\mathrm{Cl}$, at first the ciprofloxacin concentration reached a high level, prevented cells from reproduction, and in that way maintained the cell concentration at a relatively low level during the 2-hour experiment. As for chamber C4, at the very outset the ciprofloxacin concentration was low enough not to cause much effect, and so the cells grew very well. However, after one hour, more and more drug diffused from the lower chamber through the diffusion membrane to the upper one. This highly concentrated ciprofloxacin successfully inhibited cell reproduction and genetic material repairmen. As a result, the cells in chamber C4 multiplied little during the last $20 \mathrm{~min}$ of the experiment. From this 


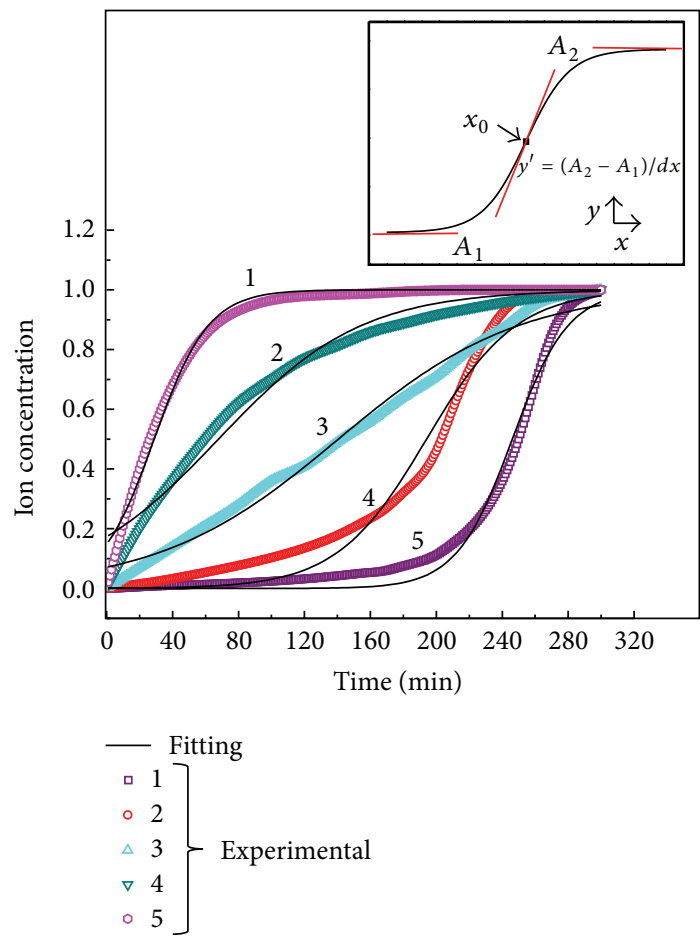

(a)

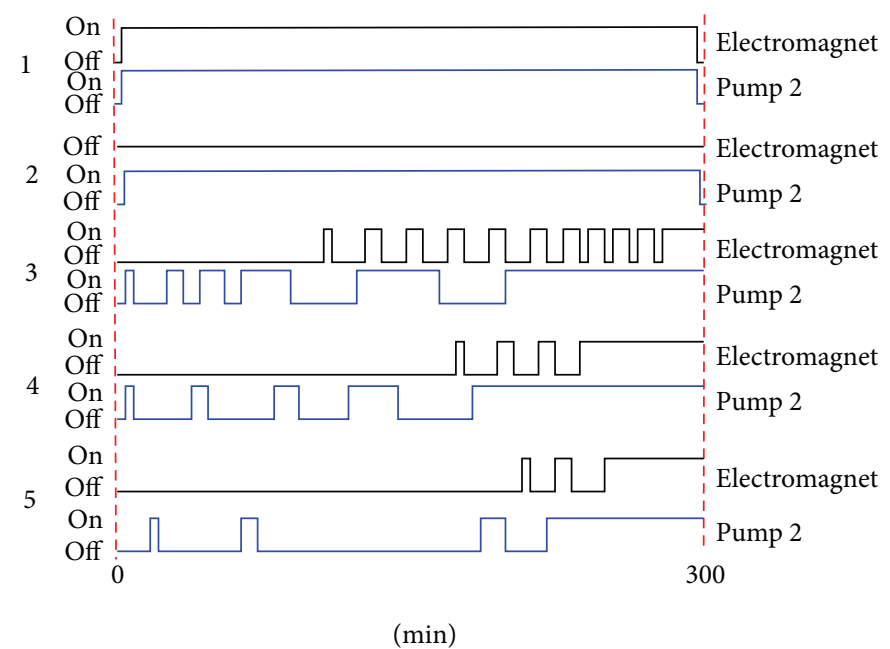

(b)

FIGURE 3: Normalized $\mathrm{NaCl}$ solution diffusion profiles. Each curve corresponds to the combined regulation from electromagnetic and syringe pump. The square-wave signal shows the working situation of electromagnet and syringe pump, respectively.

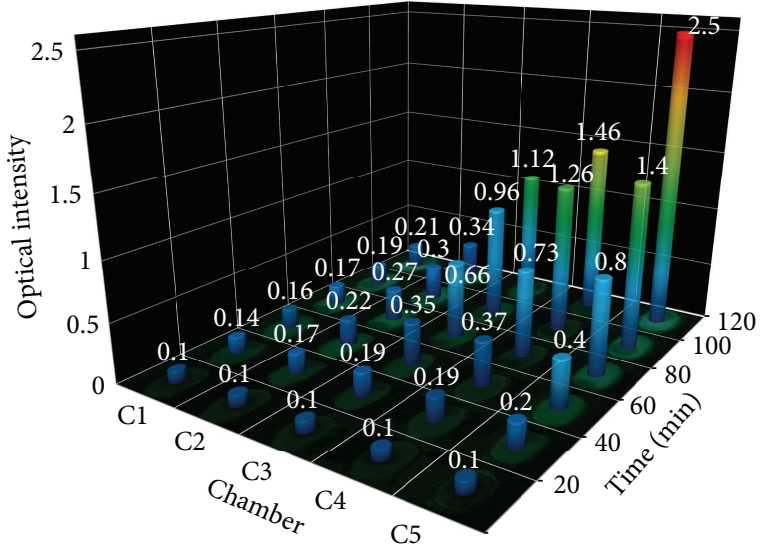

FIgURE 4: Antibacterial property of loaded fluoroquinolone ciprofloxacin in $E$. coli. The drug delivery routes of ciprofloxacin to E. coli chambers C1-C5 correspond to curves 1-5 shown in Figure 3(a), respectively. The column bars indicate the fluorescent intensity in each chamber which reflects the rough number of bacterial inside.

experiment on the antimicrobial effect, we can conclude that $E$. coli is capable of enhancing its drug resistance when ciprofloxacin increases slowly at the initial stage, even though the final dose is the same. These results agree very well with the normalized diffusion profiles in Figure 3(a). That is to say, the drug-delivery profiles indicate that treatment doses and duration times can be modulated for the desired task.

\section{Conclusions}

By utilizing magnetic nanoparticles, we successfully demonstrated a microdevice for chemical release. This kind of device has numerous potential advantages, such as being simple to fabricate and operate, high precision in chemical release, and low cost. The present research explicated the formulaic doseresponse drug-release property based on the magnetically actuated membrane. The active functionality of a microfluidic device, all of its processing digitized via actuated signals, was demonstrated in an investigation of the antibacterial property of loaded fluoroquinolone Ciprofloxacin in E. coli. The results clearly indicated the device's potential to control dosage at a satisfactory level, thereby illustrating the promise of such a controlled drug-release method. Specifically, by changing the size of the drug reservoir, the amount of medication can be accurately controlled; hence, a variety of drugs, especially sensitive or highly potent drugs, potentially can be delivered in a safe manner using the microdevice.

\section{Acknowledgments}

This paper is based on work partially supported by Award no. SA-C0040/UK-C0016, made by King Abdullah University of Science and Technology (KAUST), Saudi Arabia, Hong Kong 
RGC Grants HKUST 603608, 604710, and N_HKUST601/11, respectively.

\section{References}

[1] L. Brannon-Peppas, "Novel vaginal drug release applications," Advanced Drug Delivery Reviews, vol. 11, no. 1-2, pp. 169-177, 1993.

[2] R. Levy, M. A. Nichols, and T. W. Miller, "Encapsulated systems for controlled release and pest management," ACS Symposium Series, vol. 520, pp. 202-212, 1993.

[3] R. Langer, "Drug delivery and targeting," Nature, vol. 392, no. 6679, pp. 5-10, 1998.

[4] R. Langer, R. Siegel, and L. Brown, "Controlled release systems: some recent advances," Proceedings of the ACS Division of Polymeric Material, vol. 51, pp. 115-118, 1984.

[5] P. Gruber, M. A. Longer, and J. R. Robinson, "Some biological issues in oral, controlled drug delivery," Advanced Drug Delivery Reviews, vol. 1, pp. 1-18, 1987.

[6] M. N. V. Ravi Kumar, Handbook of Particulate Drug Delivery, vol. 2, American Scientific Publishers, 2008.

[7] P. L. Madan, "Microencapsulation II. Interfacial reactions," Drug Development and Industrial Pharmacy, vol. 4, pp. 289-304, 1978.

[8] E. Mathiowitz, D. Kline, and R. Langer, "Morphology of polyanhydride microsphere delivery systems," Scanning Microscopy, vol. 4, no. 2, pp. 329-340, 1990.

[9] A. Yu, Y. Wang, E. Barlow, and F. Caruso, "Mesoporous silica particles as templates for preparing enzyme-loaded biocompatible microcapsules," Advanced Materials, vol. 17, pp. 1737-1741, 2005.

[10] D. G. Shchukln, E. A. Ustinovich, G. B. Sukhorukov, H. Möhwald, and D. V. Sviridov, "Metallized polyelectrolyte microcapsules," Advanced Materials, vol. 17, no. 4, pp. 468-472, 2005.

[11] T. Narita, T. Yamamoto, D. Suzuki, and T. Dobashi, "Dynamics of the volume phase transition of a hydrogel membrane of a microcapsule," Langmuir, vol. 19, no. 10, pp. 4051-4054, 2003.

[12] T. Kidchob, S. Kimura, and Y. Imanishi, "Thermoresponsive release from poly $(\mathrm{Glu}(\mathrm{OMe}))$-block-poly(Sar) microcapsules with surface-grafting of poly(N-isopropylacrylamide)," Journal of Controlled Release, vol. 50, no. 1-3, pp. 205-214, 1998.

[13] A. S. Angelatos, B. Radt, and F. Caruso, "Light-responsive polyelectrolyte/gold nanoparticle microcapsules," Journal of Physical Chemistry B, vol. 109, no. 7, pp. 3071-3076, 2005.

[14] X. Yuan, K. Fischer, and W. Schärtl, "Photocleavable microcapsules built from photoreactive nanospheres," Langmuir, vol. 21, pp. 9374-9380, 2005.

[15] D. G. Shchukin, K. Köhler, and H. Möhwald, "Microcontainers with electrochemically reversible permeability," Journal of the American Chemical Society, vol. 128, pp. 4560-4561, 2006.

[16] X. Gong, S. Peng, W. Wen, P. Sheng, and W. Li, "Design and fabrication of magnetically functionalized core/shell microspheres for smart drug delivery," Advanced Functional Materials, vol. 19, no. 2, pp. 292-297, 2009.

[17] B. G. De Geest, A. M. Jonas, J. Demeester, and S. C. De Smedt, "Glucose-responsive polyelectrolyte capsules," Langmuir, vol. 22, no. 11, pp. 5070-5074, 2006.

[18] H. J. G. E. Gardeniers, R. Luttge, E. J. W. Berenschot et al., "Silicon micromachined hollow microneedles for transdermal liquid transport," Journal of Microelectromechanical Systems, vol. 12, no. 6, pp. 855-862, 2003.
[19] J.-H. Park, M. G. Allen, and M. R. Prausnitz, "Biodegradable polymer microneedles: fabrication, mechanics and transdermal drug delivery," Journal of Controlled Release, vol. 104, no. 1, pp. 51-66, 2005.

[20] M. Shikida, T. Hasada, and K. Sato, "Fabrication of a hollow needle structure by dicing, wet etching and metal deposition," Journal of Micromechanics and Microengineering, vol. 16, no. 10, pp. 2230-2239, 2006.

[21] J. Li, M. Zhang, L. Wang, W. Li, P. Sheng, and W. Wen, “Design and fabrication of microfluidic mixer from carbonyl ironPDMS composite membrane," Microfluidics and Nanofluidics, vol. 10, no. 4, pp. 919-925, 2011.

[22] L. J. Cassarett and J. Doull, Toxicology: The Basic Science of Poisons, Macmillan, 1975.

[23] J. M. Nelson, T. M. Chiller, J. H. Powers, and F. J. Angulo, "Fluoroquinolone-resistant Campylobacter species and the withdrawal of fluoroquinolones from use in poultry: a public health success story," Clinical Infectious Diseases, vol. 44, no. 7, pp. 977-980, 2007.

[24] S. Kawahara, "Chemotherapeutic agents under study," Japanese Journal of Clinical Medicine, vol. 56, no. 12, pp. 3096-3099, 1998. 

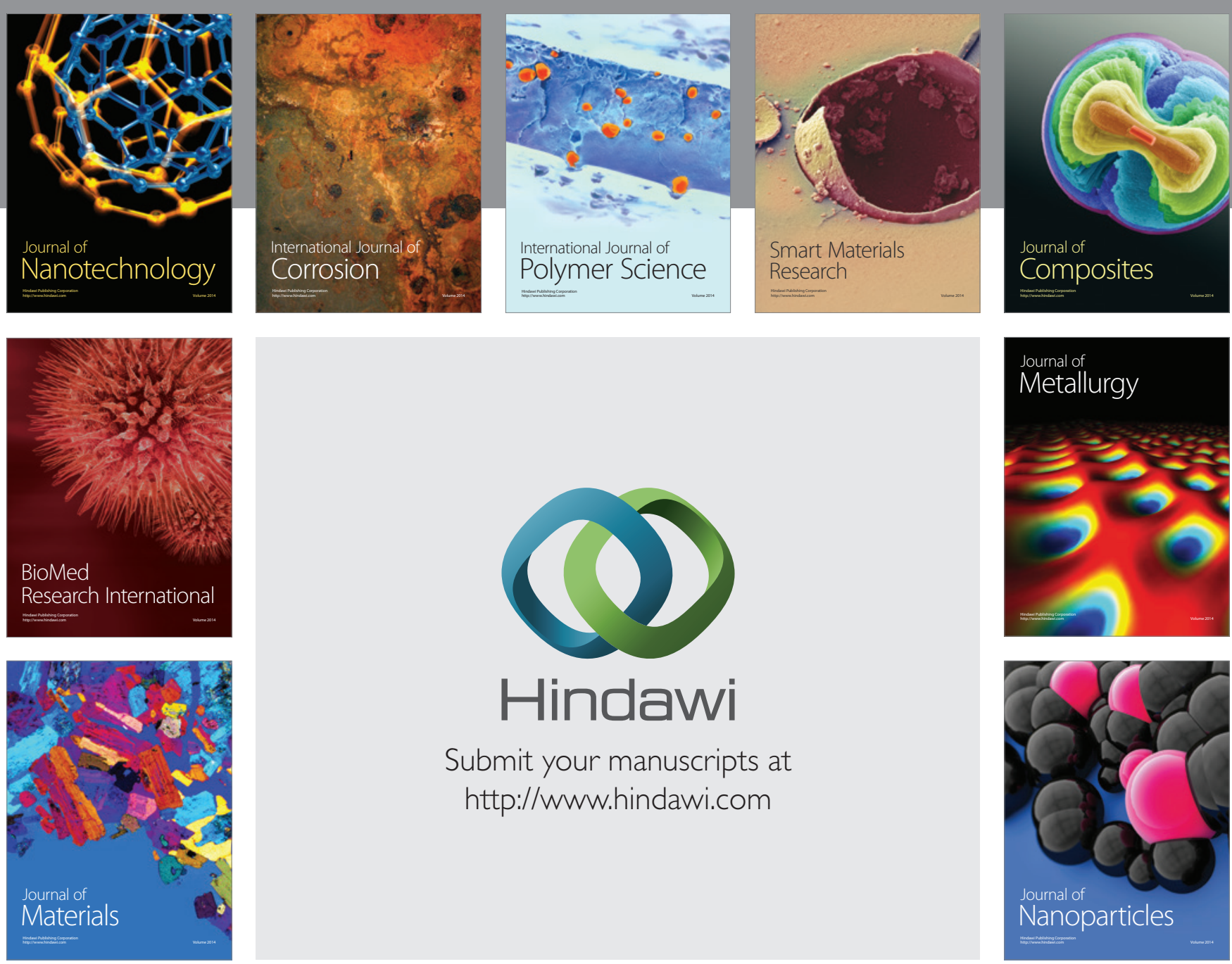

Submit your manuscripts at http://www.hindawi.com
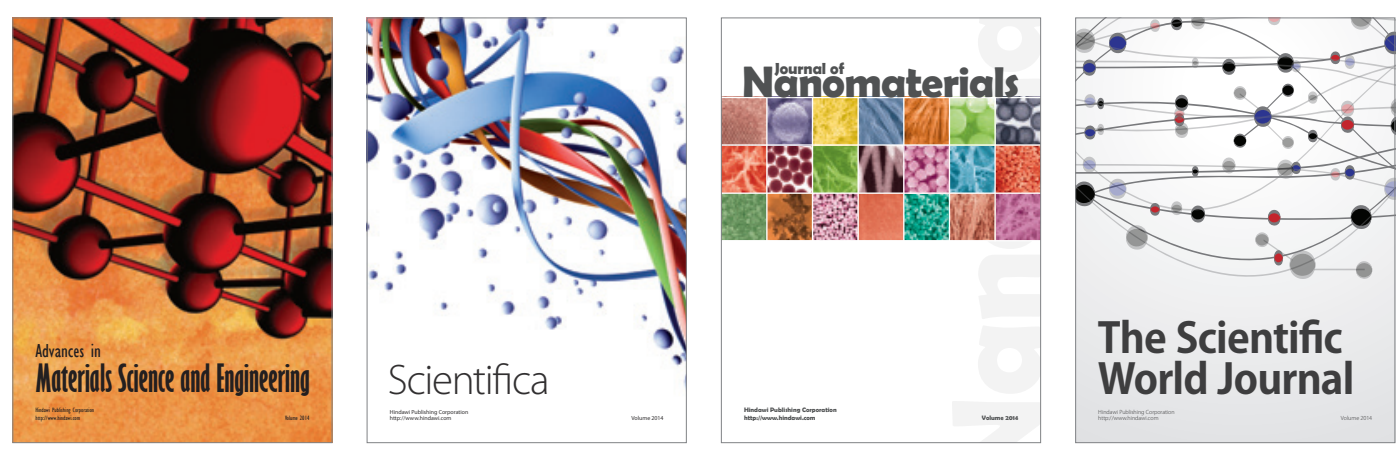

\section{The Scientific World Journal}
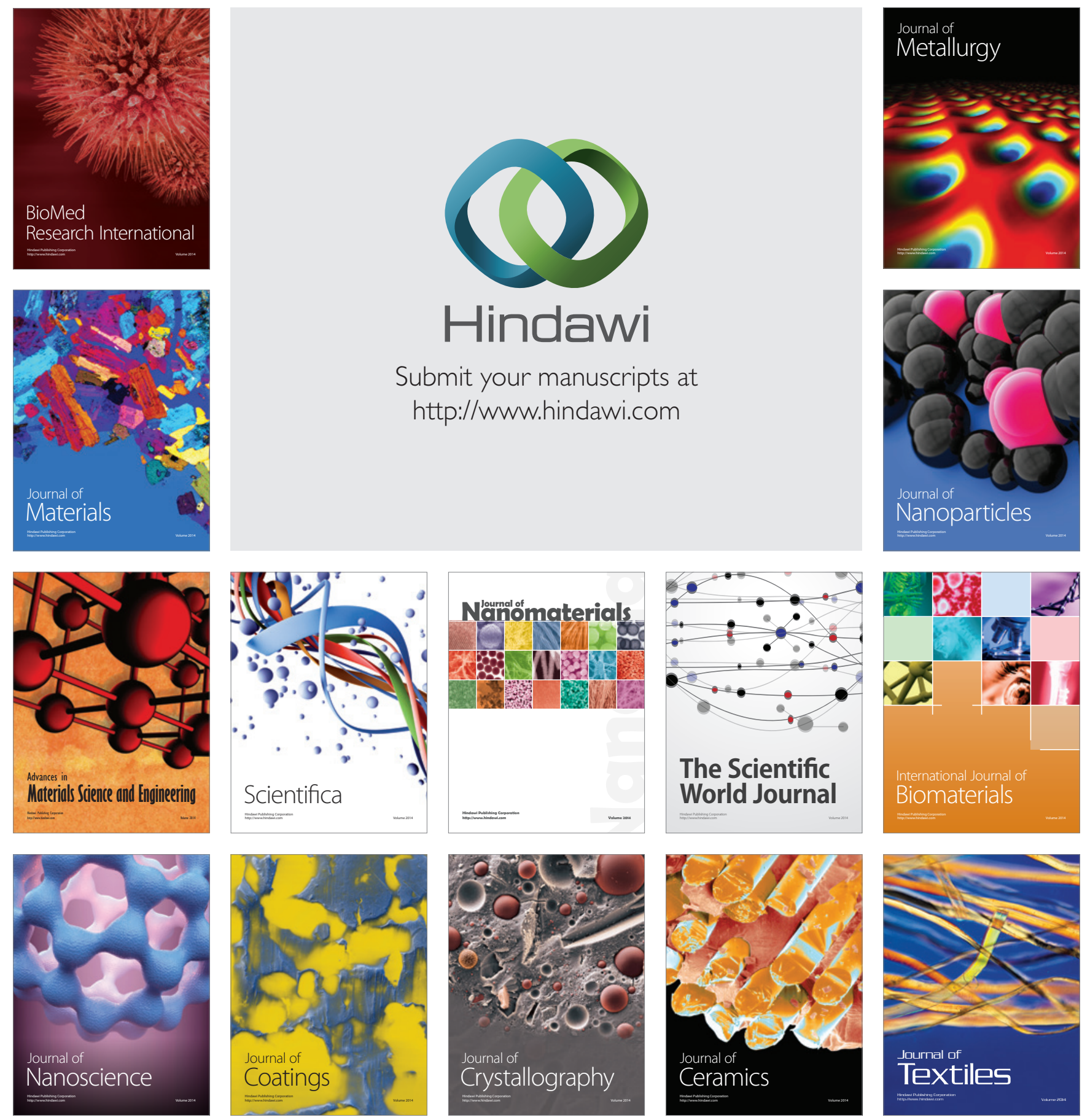